\title{
STUDY OF THE CHALCOGENIDE NONLINEAR FIBER BRAGG GRATINGS FOR ALL-OPTICAL SWITCHING
}

In this paper uniform and apodized nonlinear chalcogenide glass fiber Bragg gratings (FBG) as novel and promising devices for all-optical switching are investigated. The transmission response of these devices is discussed theoretically. The continuous wave in the investigated FBGs induces the nonlinear variation of the refractive index that causes changes in the FBG transmission characteristics. This effect can be modified depending on incident light power what is assumed to be due to the optical Kerr effect responsible for optical switching. The incident intensity thresholds of the bistable regimes were analyzed numerically to deduce possible switching properties of FBGs based on various chalcogenide glass samples. The influence of grating parameters on the optical bistability behaviour is researched. The nonlinear coupled mode theory was used for numerical investigation.

Keywords: chalcogenide glass, fiber Bragg grating, optical bistability, optical switching.

\section{Introduction}

Ultrafast optical switches are necessary parts of optical network nodes in high bit rate all-optical communication networks [1,2] including systems of wavelength division multiplexing (WDM). Optical bistability (OB) in nonlinear media is a phenomenon that can be preferably used for all-optical switching. This optical phenomenon refers to the situation in which it is possible to have two stable output transmission states associated with a single input state, depending upon the history of the input. In nonlinear switches using the optical Kerr effect, the nonlinearity must be high and the nonlinear absorption must be low [1-3]. The switching behaviour is controlled by the intensity of the input optical pulse.

Chalcogenide glasses (ChGs) containing at least one of the atoms of $\mathrm{Se}, \mathrm{S}$ or Te in their composition with the addition of As, $\mathrm{Ge}, \mathrm{Sb}$ and $\mathrm{Ga}$ can form stable nonlinear media. Due to their large transparency in the infrared region, fibers fabricated from these glasses are valuable for transmission of high power IR light. ChGs possess high third-order Kerr nonlinearity up to 1000x higher than silica glass which makes them useful for nonlinear signal processing such as nonlinear switching in optical communication systems. ChGs are highly photosensitive with photo-induced refractive index change [4-6].

Fiber Bragg gratings (FBGs) are convenient structures for large refractive index change in ChGs. FBGs are primarily applied in optical communication networks as optical filters, WDM multiplexers/demultiplexers, optical circulators, optical add-drop multiplexers, all taking advantages of simple structure, small size, and low insertion loss. Besides optical switches nonlinear FBGs can be also used as distributed reflectors [7].
In this study, we numerically investigate the threshold of the bistable behaviour in nonlinear chalcogenide FBGs. We assume uniform and apodization profiles of the FBG refractive index. Characteristics of various samples taken from [4-6, 8] of ChGs containing different chalcogens are supposed to be the nonlinear media.

\section{Theory}

FBGs can be fabricated by the exposing photosensitive optical fiber with intensive ultraviolet light. It causes the variations of the refractive index along the FBG length $L$ periodically (Fig. 1).

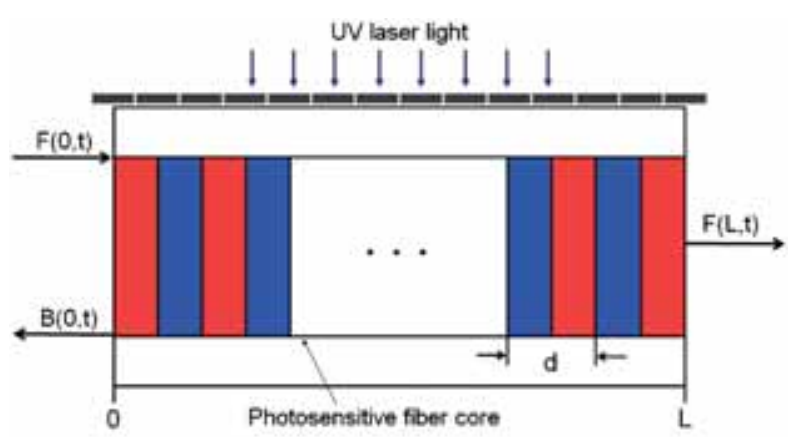

Fig. 1 Schematic diagram of FBG, where $F$ and $B$ represent envelopes of the forward and backward travelling waves respectively and $d$ is the FBG period, $L$ is the grating length

\footnotetext{
* Eliska Jurisova, Jarmila Mullerova

Department of Engineering Fundamentals, Faculty of Electrical Engineering, University of Zilina, Slovakia,

E-mail: jurisova@lm.uniza.sk
} 
The dependence of the refractive index on the position in the FBG (supposing the radiation is propagating in the $z$ direction along the axis of the fiber) can be written as [9-11]:

$$
n(z)=n_{e f f}+V_{n} g(z) v\left(\cos \frac{2 \pi z}{d}\right)+n_{2}|\vec{E}(z)|^{2}
$$

Here $n_{\text {eff }}$ is the effective (average) refractive index, $V_{\mathrm{n}}$ is the depth of the modulation of the refractive index, $g(z)$ is an apodization function, $v$ introduces the fringe visibility, $n_{2}$ is nonlinear refractive index and $\vec{E}(z)$ is the electric field.

Apodization is a well-known technique in which the refractive index is graded also in the direction perpendicular to the grating length. This technique can effectively remove the undesirable sidelobes in the reflection/transmission spectra of linear FBGs used as WDM multiplexers/demultiplexers or optical filters. The common apodization profiles are in Table 1 [11-12].

The light propagation in the nonlinear gratings is governed by the set of nonlinear coupled mode equations (NCMEs) [9-11, 13]:

$$
\begin{aligned}
& \frac{\partial F}{\partial z}=i\left[\delta F+\kappa B+\gamma|F|^{2} F+2 \gamma|B|^{2} F\right]-\frac{c}{n_{\text {eff }}} \frac{\partial F}{\partial t} \\
& \frac{\partial B}{\partial z}=-i\left[\delta B+\kappa F+\gamma|B|^{2} B+2 \gamma|F|^{2} B\right]-\frac{c}{n_{e f f}} \frac{\partial B}{\partial t}(
\end{aligned}
$$

$F$ and $B$ are the amplitudes of the forward and backward modes, which determine the electric field inside the grating. $|F|^{2}$ and $|B|^{2}$ are proportional to the intensity of light. Coupling coefficient $\kappa$ is defined as

$$
\kappa=\frac{\pi V_{n} v g}{\lambda_{\text {Bragg }}}
$$

where $\lambda_{\text {Bragg }}$ is the Bragg (central) wavelength defined as $\lambda_{\text {Bragg }}=$ $=2 \pi_{\text {neff }} \cdot \delta$ represents the detuning between $\lambda_{\text {Bragg }}$ and the carrier wavelength $\lambda$ :

$$
\delta=2 \pi n_{e f f}\left(\frac{1}{\lambda}-\frac{1}{\lambda_{\text {Bragg }}}\right)
$$

The parameter $\gamma$ denotes the nonlinear Kerr effect coefficient:

$$
\gamma=\frac{2 \pi n_{2}}{\lambda_{\text {Bragg }}}
$$

In linear regime $\gamma=0$.
For the solving of NCMEs finite difference method (FDM) was used in order to obtain the relationship of the incident intensity and the transmittance of FBG. This method requires initial value points: $F(z=0, \mathrm{t})=A(t), F(z, 0)=0, B(z, 0)=0$. As initial conditions we assumed the zero amplitude of the backward wave: $B(z=L, t)=$ $=0$.

OB occurs when the coupling coefficient, detuning parameter and nonlinearity coefficient match some conditions. This is significant to realize nonlinear switching in FBGs at the Bragg wavelength $[4,14]$. The transmittance is defined as the ratio of the output and the incident light intensities.

\section{Results and Discussion}

In this section, we numerically simulate the bistable behaviour in nonlinear FBGs for different samples of ChGs the characteristics of which are depicted in Table 2. These experimental data are taken from [4-6, 8]. As it is supposed to apply nonlinear FBG switches mainly in the optical communication systems, it is important to set the investigation to the $3^{\text {rd }}$ transmission spectral window ( $\mathrm{C}$ band, around $1550 \mathrm{~nm}$ ). Therefore we assume the propagating wave centred at the Bragg wavelength of $1550 \mathrm{~nm}$. The investigated FBGs have parameters of the following values: $L=10 \mathrm{~mm}$ and $V_{\mathrm{n}}=0.0001$.

Parameters for selected $\mathrm{ChGs}$ samples taken

Table 2 from the literature $[4-6,8]$.

\begin{tabular}{|c|c|c|}
\hline Chalcogenide glass & $n_{\text {eff }}$ & $n_{2} \times 10^{-14}\left[\mathrm{~cm}^{2} / \mathrm{W}\right]$ \\
\hline $\mathrm{As}_{2} \mathrm{~S}_{3}$ & 2.45 & 2.6 \\
\hline $\mathrm{As}_{2} \mathrm{Se}_{3}$ & 2.81 & 14 \\
\hline $\mathrm{Ge}_{10} \mathrm{As}_{10} \mathrm{Se}_{80}$ & 2.58 & 6.8 \\
\hline $\mathrm{Ge}_{10} \mathrm{As}_{10} \mathrm{Se}_{70} \mathrm{Te}_{10}$ & 2.74 & 8.4 \\
\hline $\mathrm{Ge}_{10} \mathrm{As}_{10} \mathrm{Se}_{60} \mathrm{Te}_{20}$ & 2.90 & 13.4 \\
\hline $\mathrm{Ge}_{5} \mathrm{As}_{30} \mathrm{Se}_{65}$ & 2.72 & 6.2 \\
\hline $\mathrm{Ge}_{15} \mathrm{As}_{34} \mathrm{Se}_{51}$ & 2.64 & 3.9 \\
\hline $\mathrm{Ge}_{35} \mathrm{As}_{15} \mathrm{Se}_{50}$ & 2.63 & 3.6 \\
\hline
\end{tabular}

We suppose the stationary state of nonlinear systems. Then NCMEs (2) take the form

\begin{tabular}{|c|c|c|}
\hline Gaussian profile & Sinc profile & Raised-cosine profile \\
\hline$g(z)=\exp \left(G\left(\frac{z-\frac{L}{2}}{L}\right)^{2}\right)$ & $g(z)=\frac{\sin (x)}{x}, x=\frac{2 \cdot \pi}{L}\left(z-\frac{L}{2}\right)$ & $g(z)=\frac{1}{2}\left[1+\cos \left(\frac{\pi}{L}\left(z-\frac{L}{2}\right)\right)\right]$ \\
\hline
\end{tabular}




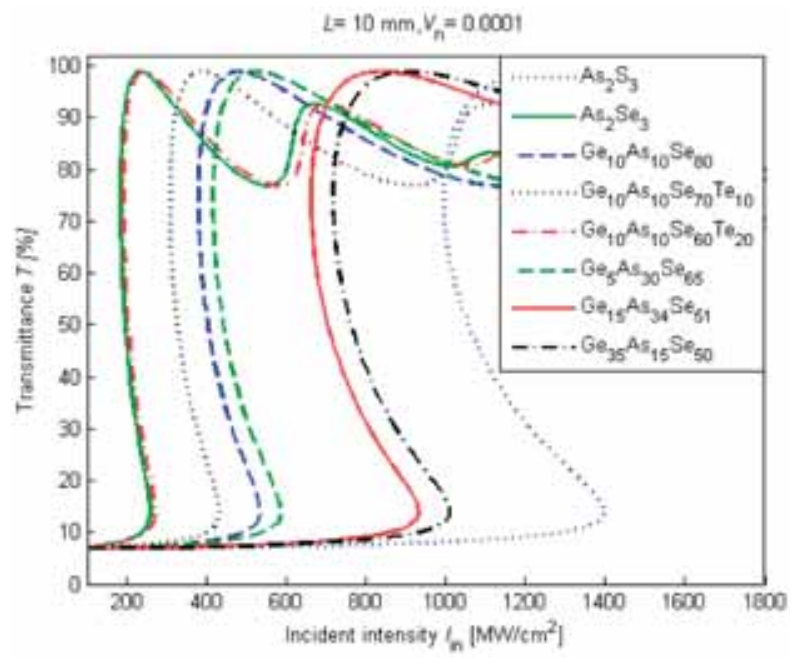

a) $\delta=0$

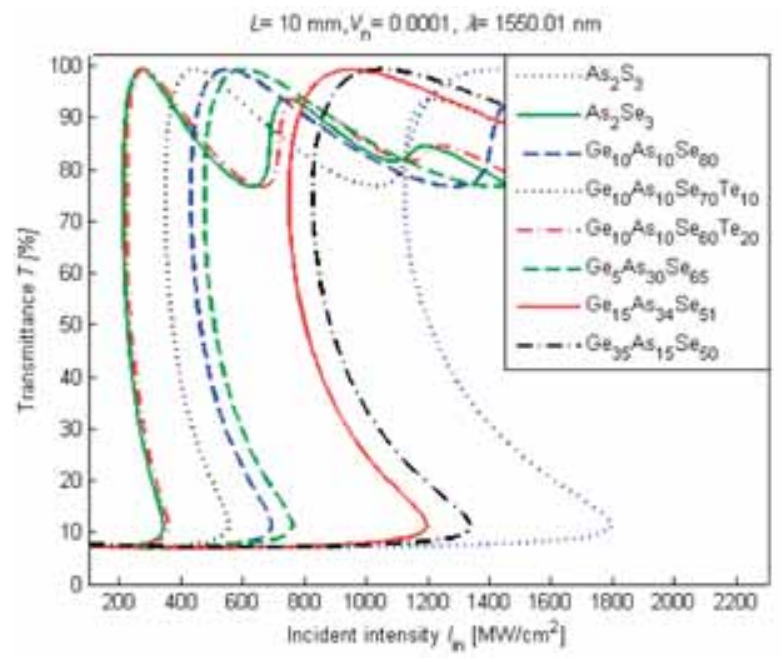

b) $\delta=-0.73489 \mathrm{~cm}^{-1}$

Fig. 2 The optical bistability curves for nonlinear FBGs based on ChGs. The values of their refractive and nonlinear index are in Table 2

$$
\begin{aligned}
& \frac{\partial F}{\partial z}=i\left[\kappa B+\delta F+\gamma\left(|F|^{2}+2|B|^{2}\right) F\right] \\
& \frac{\partial B}{\partial z}=-i\left[\kappa F+\delta B+\gamma\left(|B|^{2}+2|F|^{2}\right) B\right]
\end{aligned}
$$

First, we present the results for FBGs supposed to be made from eight samples from Table 2 for the detuning parameter $\delta=0$ (Fig. 2a) ) and $\delta=-0.73489 \mathrm{~cm}^{-1}$ (Fig. 2b) ). We see that all dependences manifest optical bistability (even multistability at higher input intensities). From Fig. 2 it is clear that the incident intensity required for the optical switching is minimal for $\mathrm{As}_{2} \mathrm{Se}_{3}$ and maximal for $\mathrm{As}_{2} \mathrm{~S}_{3}$. Other suitable samples requiring moderate switching intensities seem to be ChGs with tellurium. As the samples differ by refractive indices, their important impact on the switching intensity can be deduced. As the nonlinear index increases, the incident intensity needed for switching decreases.

\subsection{Results for the Chalcogenide glass $\mathrm{As}_{2} \mathrm{Se}_{3}$}

In the next simulations we concentrate only on the sample of $\mathrm{As}_{2} \mathrm{Se}_{3}$ requiring the minimal switching intensity of all studied samples. In Fig. 3 the typical bistability curve only for this sample is depicted while the simulation parameters are the same as in Fig. 2a) and 2b).

Fig. 3 represents the phenomenon of $\mathrm{OB}$ as the following change of the transmittance $(T)$ as a result of the incident intensity $\left(I_{\text {in }}\right)$. As $I_{\text {in }}$ increases from zero, $T$ follows the lower branch of the curve until Iin reaches the switching point (switch-on threshold), where the output makes a sudden jump to the upper branch. When $I_{\text {in }}$ drops from high intensities, $T$ can be brought back to the lower branch (switch-off threshold).The state of a linear increase of $I_{\text {in }}$ is termed as the "OFF" state and the state of a sharp rise in Iin for $I_{\mathrm{in}}>I_{\mathrm{A}}$ is called the "ON" state. The difference of the transmission between "ON" and "OFF" states is the so-called on-off contrast. It indicates the transmittance interval $\left(T_{\mathrm{A}}-T_{\mathrm{B}}\right)$ of the optical switching called the on-off contrast. The width of the Sshaped curve (the so-called hysteresis width, w) is defined as the difference between the switch-on (point $I_{\mathrm{B}}$ ) and switch-off (point $I_{\mathrm{A}}$ ) intensity thresholds.

It is clear that the switch-on intensity and the width of the hysteresis loop of $\mathrm{OB}$ increase with increasing the carrier wavelength (comparing Fig. 3a) and 3b)). The hysteresis width $w$ for the case of $\delta=0$ is $w \sim 72 \mathrm{MW} / \mathrm{cm}^{2}$ while for $\delta=-0.73489 \mathrm{~cm}^{-1}$ it is $w \sim 127 \mathrm{MW} / \mathrm{cm}^{2}$.

Next we present the numerical results for the influence of the Bragg wavelength on OB behaviour (Fig. 4). The carrier wavelength is suggested to be $\lambda=1550.03 \mathrm{~nm}$. The increasing of this wavelength causes the increasing in the hysteresis width and in the switch-on intensity as well. Results in Fig. 4b) of Bragg wavelength variations are plotted for $\delta=-2.2046 \mathrm{~cm}^{-1}$. It is clear that in the all cases OB occurs. Therefore FBGs can be designed as optical switches for specified WDM channels.

\subsection{The Impact of the Apodization and Parameters of FBG}

It is interesting to compare the nonlinear switching characteristics for uniform and apodized FBGs. The results for the uniform FBG and several apodized FBGs are presented in Fig. 5. The simulations show that the OB curve is present for all investigated index profiles, but the S-shape for the curves of $\mathrm{OB}$, the values for 


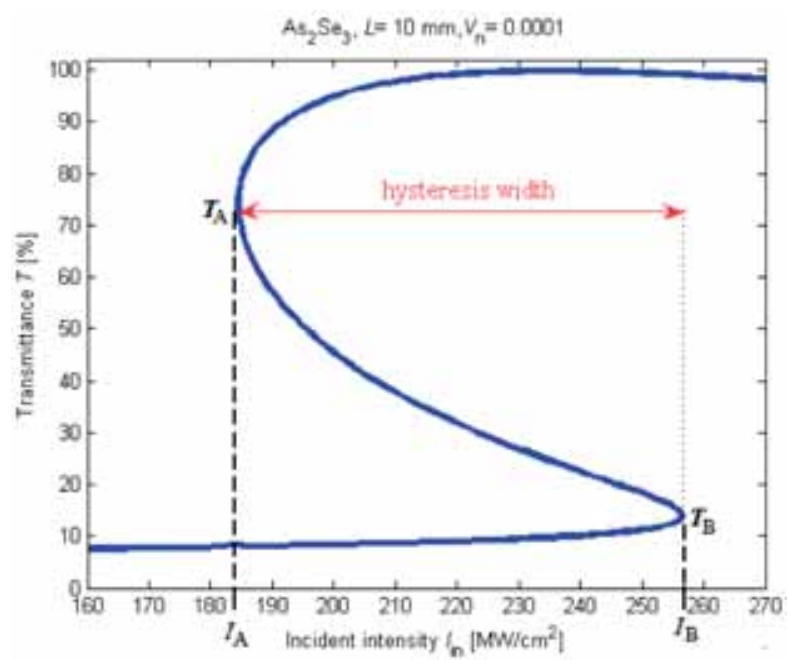

a) $\delta=0$

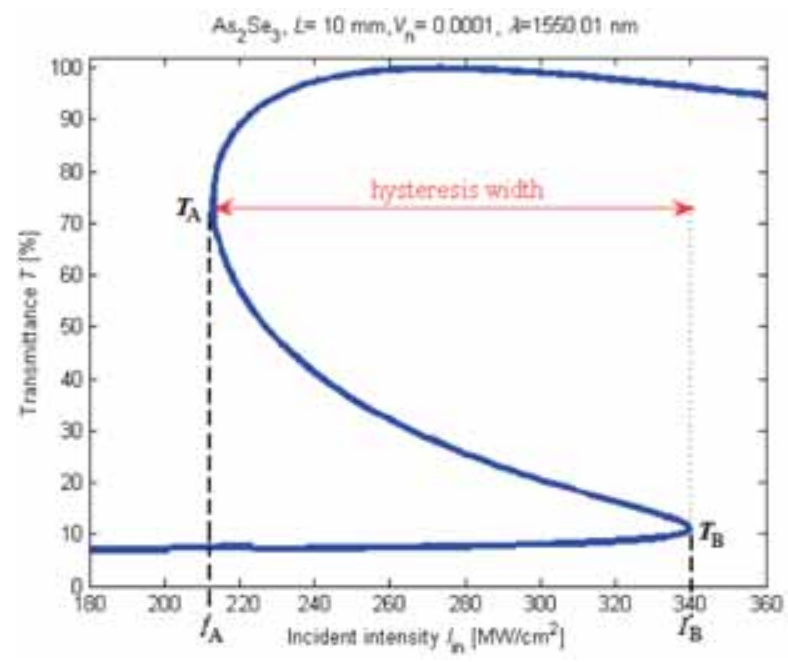

b) $\delta=-0.73489 \mathrm{~cm}^{-1}$

Fig. 3 The optical bistability curve for $\mathrm{As}_{2} \mathrm{Se}_{3}$

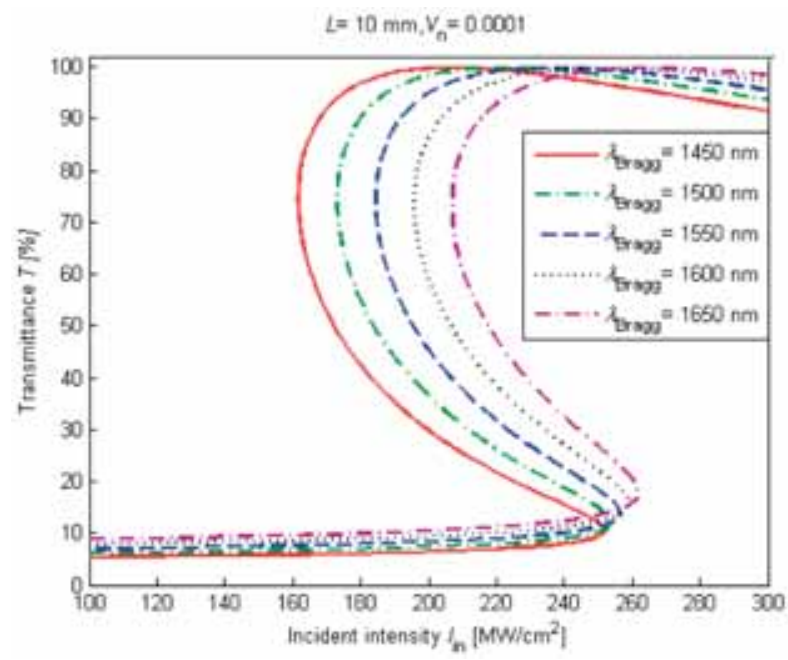

a) $\delta=0$

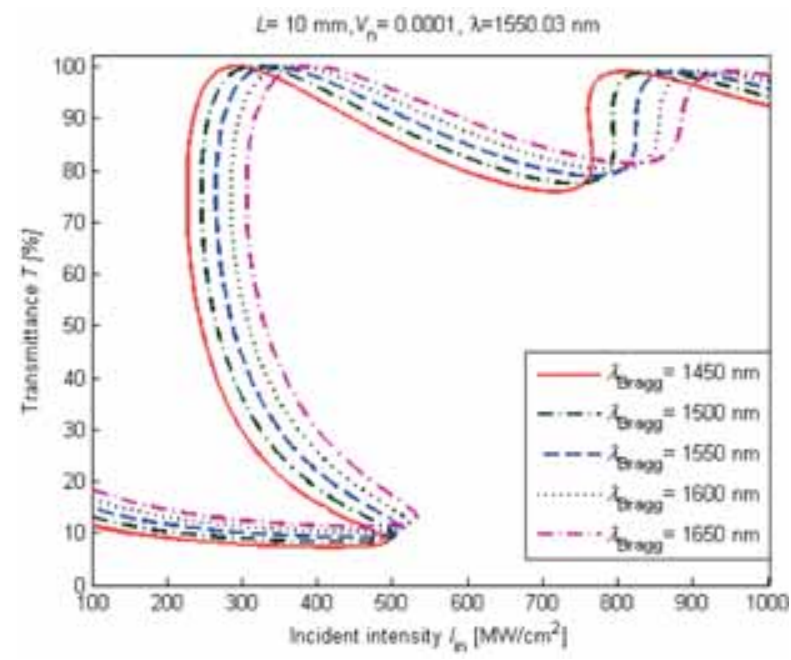

b) $\delta=-2.2046 \mathrm{~cm}^{-1}$

Fig. 4 The optical bistability curve for $\mathrm{As}_{2} \mathrm{Se}_{3}$ influenced by the change of $\mathrm{\lambda Bragg}$

the transmittance, incident intensity and hysteresis width differ. The on-off contrast is higher for uniform gratings. We see that the apodization reduces the on-off contrast. Besides this we observe that apodized FBGs show the hysteresis width reduced in comparison with uniform FBGs. Both can be detrimental for optical switching that could fail or become accidental in these cases. Therefore we decided to alter some FBG parameters in wider intervals to study how they affect the switching conditions.

The investigation of the influence of the grating length $L$ is depicted in Fig. 6. For the uniform FBG the length should be at least $7 \mathrm{~mm}$ to exhibit a slight bistability while for apodized FBGs of this length the bistability does not occur. Increasing on-off contrasts and hysteresis widths are observed with increasing $L$ which is promising for optical switching. However, we found out that the on-off contrasts for sinc and raised-cosine apodization profiles are much smaller than those for Gaussian and uniform FBGs.

To improve the results we recommend optimizing the depth of the modulation of the refractive index $V_{\mathrm{n}}$ and/or the grating length $L$. Fig. 7 shows that higher $V_{\mathrm{n}}$ improves the on-off contrast 


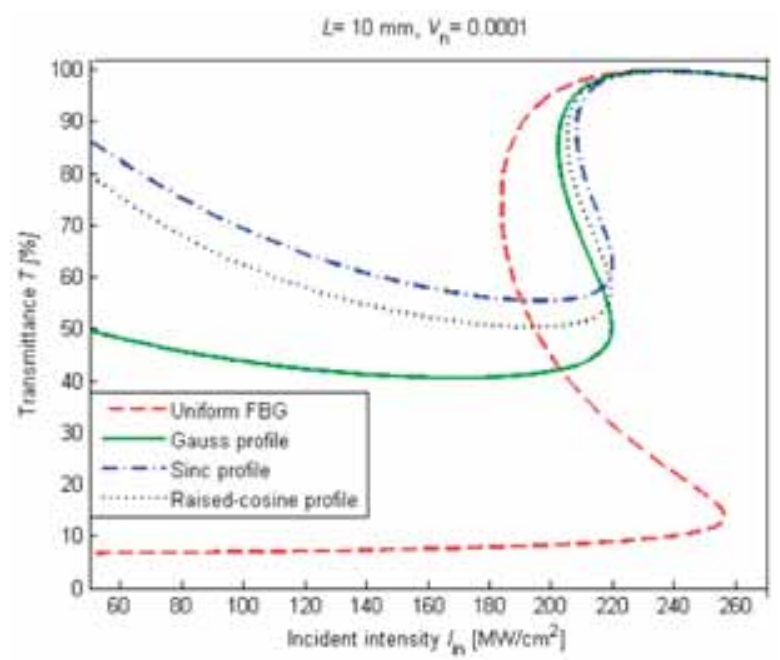

a) $\delta=0$

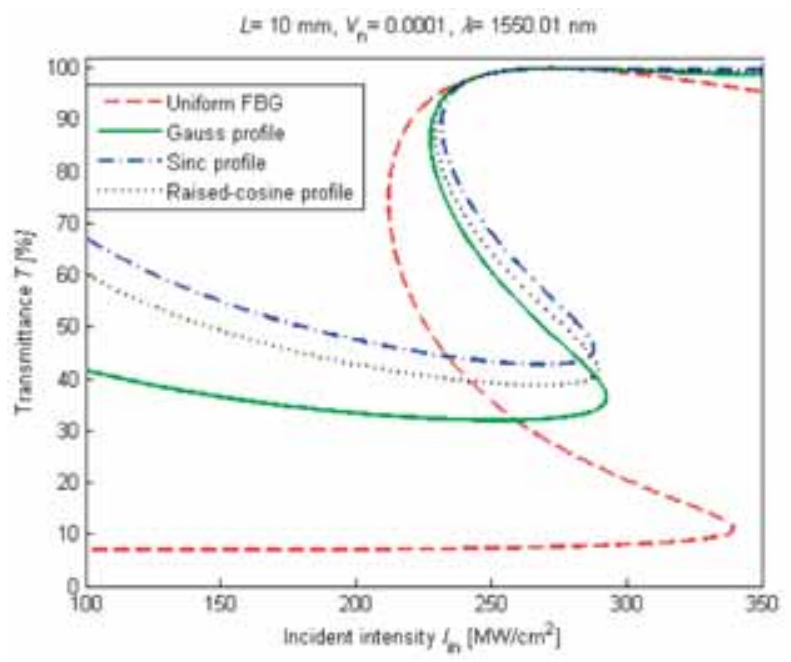

b) $\delta=-0.73489 \mathrm{~cm}^{-1}$

Fig. 5 Results comparison for $\mathrm{As}_{2} \mathrm{Se}_{3}$ for uniform $\mathrm{FBG}$ and apodization $\mathrm{FBG}$

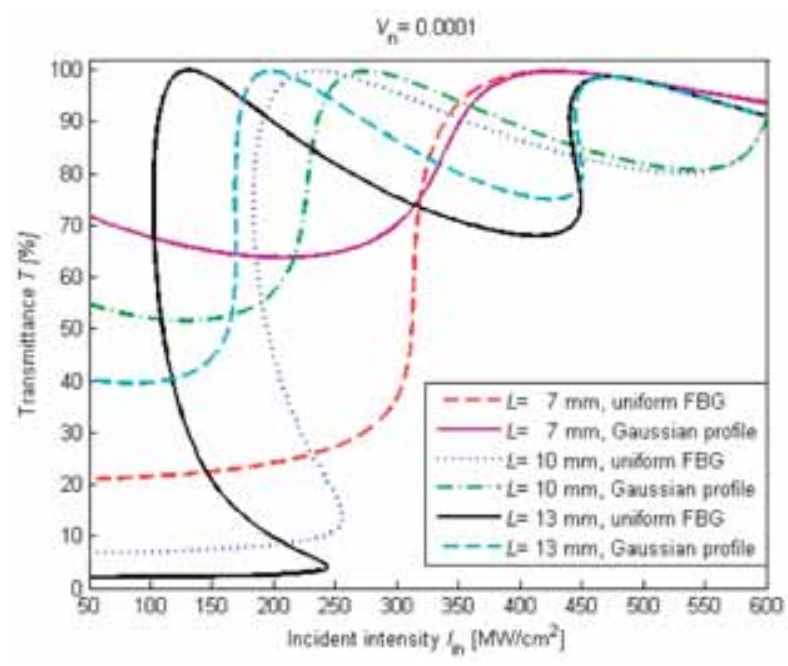

a) uniform and Gaussian apodized FBG

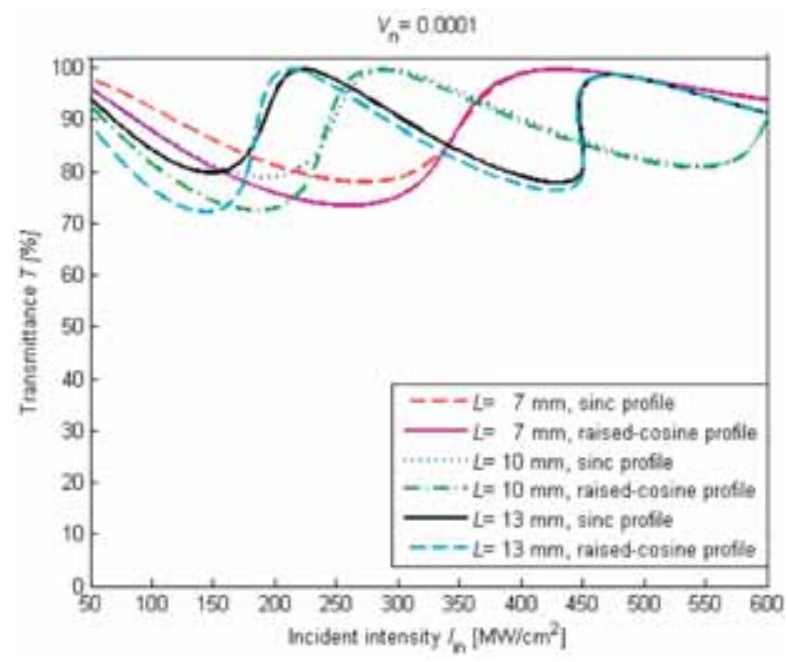

b) sinc and raised-cosine apodized $F B G$

Fig. 6 Results comparison for $\mathrm{As}_{2} \mathrm{Se}_{3}$ of the uniform and apodized $\mathrm{FBG}$ by the change of $L$

of both uniform and Gaussian apodized FBGs. The changes are similar as those mapping the influence of the grating length.

For sinc and raised-cosine apodized FBGs, higher grating length is necessary to achieve desirable on-off contrasts and hysteresis widths. Therefore we simulate the influence of the depth of modulation at the grating length of $L=20 \mathrm{~mm}$ (Fig. 8) believing to achieve better results as in Fig. 6b). We see that with increasing $V_{\mathrm{n}}$ the on-off contrast is improved in comparison with data in Fig. 6b) although still not so pronounced as for uniform and Gaussian apodized FBGs.

\section{Conclusion}

In this paper we numerically demonstrated the optical bistability behaviour of the uniform and apodized chalcogenide nonlinear FBGs. The NCMEs were solved with FDM for the obtaining the switching threshold for our investigation. We found out the importance of FBG parameters as the FBG length, the depth of modulation of the refractive index and the Bragg wavelength, because they could have considerable influence on the shape of the hysteresis curve, the bistable threshold, the hysteresis width and the on-off 


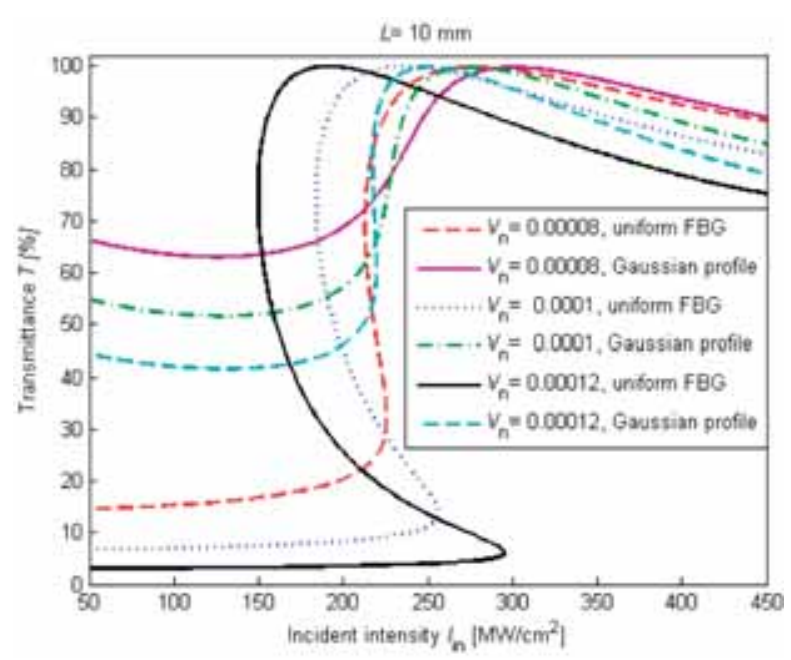

Fig. $7 O B$ in uniform and Gaussian apodized FBG, influence of varied $V_{n}$

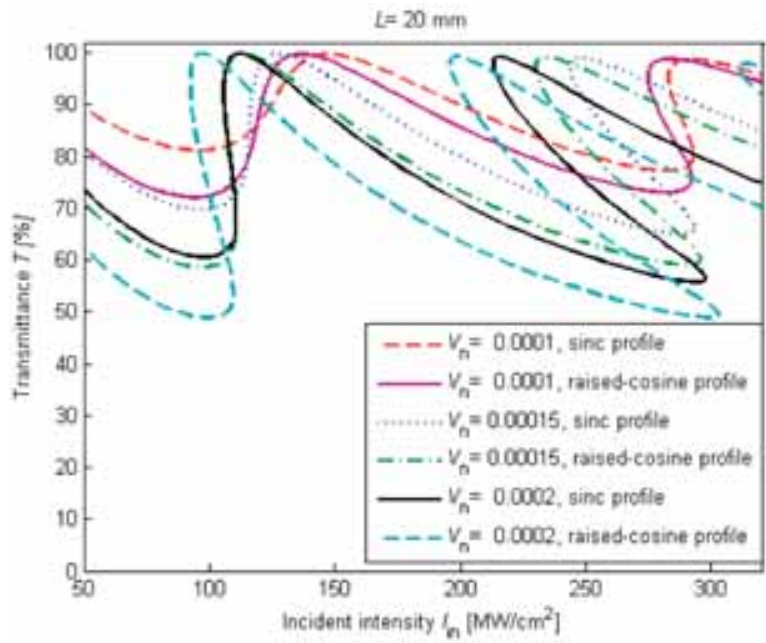

Fig. $8 O B$ in sinc- and raised-cosine apodized $F B G$, influence of varied $V_{n}$

This work was partly supported by the Slovak Grant Agency under project No. 1/0411/10. contrast. Uniform FBGs appeared to be better for all-optical switching having higher on-off contrasts than the apodized FBGs.

Acknowledgement

\section{References}

[1] WINFUL, H. G., MARBURGER, S. D., GARMIRE, E.: Theory of Bistability in Nonlinear Distributed Feedback Structures, Appl. Phys. Lett., 35 (5), 1979

[2] DADO, M., KRAJCI, S., DUBOVAN, J., SABOL, D.: Numerical Investigation of Optical Burst Switching. Communication - Scientific Letters of the University of Zilina, pp. 20-24, No. 2, 2008

[3] YOSIA, Y., PING, S.: Optical Bistability in Periodic Media with Third-, Fifth-, and Seventh-Order Nonlinearities, J. of Light Wave Technology, vol. 25, 3875-882, 2007

[4] Zakery, A. Elliot, S. R.: Optical Nonlinearities in Chalcogenide Glasses and their Applications, Springer Verlag Berlin Heidelberg, 2007

[5] PRASAD, A: Properties of $G e_{x} A s_{y} S e_{1-x-y}$ Glasses for All-optical Signal Processing, Optics Express, vol. 16, No. 4, 2008

[6] HARBOLD, J. M. et al.: Highly nonlinear As-S-Se Glasses for All-optical Switching, Optics Letters, 27(2) , pp. 119-21, 2002

[7] CARVALHO, J. C. C., et al.: A New Acceleration Technique for the Design of Fiber Gratings, Optical Society of America, Optics Express, vol. 14, No. 22, 10715-10725, 2006

[8] CHERUKULAPPURATH, S. et al.: Linear and Nonlinear Optical Characterization of Tellurium Based Chalcogenide Glasses, Opt. Communication 242, pp. 313-319, 2004

[9] PENG, Y. et al.: Study on Characteristics of Optical Bistable Devices Based on Fiber Bragg Grating, OSA/ACP, SPIE-OSA-IEEE, WL12, vol. 7630, 2009

[10] LEE, H., AGRAWAL, G. P: Nonlinear Switching of Optical Pulses in Fiber Bragg Gratings, IEEE J. of Quantum Electronics, vol. 39, No. 3, 2003

[11] MAHRAN, O. et al.: Reflectivity of Nonlinear Apodized Chirped Fiber Bragg Grating under Water, J. of Applied Science Research, 5(10), pp. 1604-1610, 2009

[12] UGALE, S., MISHRA, V.: Fiber Bragg Grating Modelling, Characterization and Optimization with Different Index Profiles, Intern. J. of Engineering Science and Technology, vol. 2 (9), pp. 4463-4468, 2010

[13] STERKE, C. M., JACKSON, K. R., ROBERT, B. D.: Nonlinear Coupled Mode Equations on a Finite Interval: A Numerical Procedure, J. Opt. Soc. Am B, vol. 8, No. 2, 403-412, 1991

[14] YOSIA, Y., PING, S.: Optical Bistability in Periodic Media with Third-, Fifth-, and Seventh-Order Nonlinearities, J. of Lightwave Technology, vol. 25, pp. 3875-882, 2007. 